\title{
Short Note on the Riemann Hypothesis
}

\author{
Frank Vega
}

CopSonic, 1471 Route de Saint-Nauphary 82000 Montauban, France

\begin{abstract}
Robin criterion states that the Riemann Hypothesis is true if and only if the inequality $\sigma(n)<e^{\gamma} \times$ $n \times \log \log n$ holds for all $n>5040$, where $\sigma(n)$ is the sum-of-divisors function and $\gamma \approx 0.57721$ is the Euler-Mascheroni constant. This is known as the Robin inequality. We know that the Robin inequality is true for all $n>5040$ which are not divisible by 2 . In addition, we prove the Robin inequality is true for all $n>5040$ which are divisible by 2. In this way, we show the Robin inequality is true for all $n>5040$ and thus, the Riemann Hypothesis is true.
\end{abstract}

Keywords: Riemann hypothesis, Robin inequality, sum-of-divisors function, prime numbers 2000 MSC: 11M26, 11A41, 11A25

\section{Results}

In mathematics, the Riemann Hypothesis is a conjecture that the Riemann zeta function has its zeros only at the negative even integers and complex numbers with real part $\frac{1}{2}$ [1]. As usual $\sigma(n)$ is the sum-of-divisors function of $n$ [2]:

$$
\sum_{d \mid n} d
$$

where $d \mid n$ means the integer $d$ divides to $n$. Define $f(n)$ to be $\frac{\sigma(n)}{n}$. Say Robins $(n)$ holds provided

$$
f(n)<e^{\gamma} \times \log \log n .
$$

The constant $\gamma \approx 0.57721$ is the Euler-Mascheroni constant and $\log$ is the natural logarithm. The importance of this property is:

Theorem 1.1. Robins $(n)$ holds for all $n>5040$ if and only if the Riemann Hypothesis is true [1].

It is known that Robins $(n)$ holds for many classes of numbers $n$.

Theorem 1.2. Robins $(n)$ holds for all $n>5040$ that are not divisible by 2 [2].

In addition, we know that:

Theorem 1.3. Robins $(n)$ holds for all $10^{10^{10}} \geq n>5040$ [3].

Email address: vega.frank@gmail.com (Frank Vega) 
Let $h(n)$ be defined as

$$
h(n)=\prod_{q \mid n} \frac{q}{q-1}
$$

These are known results:

Theorem 1.4. [2]. For $n>1$ :

$$
f(n)<h(n) .
$$

Theorem 1.5. [4]. For $n \geq 3$ :

$$
h(n)<e^{\gamma} \times \log \log n+\frac{2.50637}{\log \log n} .
$$

Let's prove our main result:

Theorem 1.6. Robins $(n)$ holds for all $n>5040$ that are divisible by 2 .

Proof. Let's assume that $n>5040$ is divisible by 2 . We have that

$$
f(n) \leq f(2) \times f\left(\frac{n}{2}\right)
$$

since the function $f(n)$ is submultiplicative (that is $f(q \times r) \leq f(q) \times(r))$ [2]. We use that theorem 1.4 to show that

$$
f(2) \times f\left(\frac{n}{2}\right) \leq f(2) \times h\left(\frac{n}{2}\right)=\frac{f(2)}{h(2)} \times h(n)=\frac{3}{4} \times h(n)
$$

since $f(2)=\frac{3}{2}$ and $h(2)=2$. According to the theorem 1.5 , we obtain that

$$
f(n) \leq \frac{3}{4} \times h(n)<\frac{3}{4} \times\left(e^{\gamma} \times \log \log n+\frac{2.50637}{\log \log n}\right) .
$$

Hence, it is enough to prove that

$$
\frac{3}{4} \times\left(e^{\gamma} \times \log \log n+\frac{2.50637}{\log \log n}\right) \leq e^{\gamma} \times \log \log n
$$

which is equivalent to

$$
\frac{3}{4} \times\left(1+\frac{2.50637}{e^{\gamma} \times(\log \log n)^{2}}\right) \leq 1
$$

after of dividing the both sides of the inequality by $e^{\gamma} \times \log \log n$. We know that Robins $(n)$ holds for all $10^{10^{10}} \geq n>5040$ due to the theorem 1.3. Consequently, we would have that

$$
\left(\frac{3}{4}+\frac{3}{4} \times \frac{2.50637}{e^{\gamma} \times(\log \log n)^{2}}\right)<\left(\frac{3}{4}+\frac{3}{4} \times \frac{2.50637}{e^{\gamma} \times\left(\log \log 10^{10^{10}}\right)^{2}}\right)
$$

for $n>10^{10^{10}}$. In this way, it is enough to show that

$$
\left(\frac{3}{4}+\frac{3}{4} \times \frac{2.50637}{e^{\gamma} \times\left(\log \log 10^{10^{10}}\right)^{2}}\right) \leq 1
$$


which is the same as

$$
\frac{3}{4} \times \frac{2.50637}{e^{\gamma} \times\left(\log \log 10^{10^{10}}\right)^{2}} \leq \frac{1}{4}
$$

that is equal to

$$
\frac{3 \times 2.50637}{e^{\gamma} \times\left(\log \log 10^{10^{10}}\right)^{2}} \leq 1
$$

after of multiplying by 4 . Finally, we need to prove that

$$
3 \times 2.50637 \leq e^{\gamma} \times\left(\log \log 10^{10^{10}}\right)^{2}
$$

which is trivially true and therefore, the proof is complete.

This result implies the following consequences:

Theorem 1.7. Robins $(n)$ holds for all $n>5040$.

Proof. This is a direct consequence of theorems 1.2 and 1.6

Theorem 1.8. The Riemann Hypothesis is true

Proof. This is true because of the theorems 1.1 and 1.7.

\section{References}

[1] G. Robin, Grandes valeurs de la fonction somme des diviseurs et hypothèse de Riemann, J. Math. pures appl 63 (2) (1984) $187-213$

[2] Y. Choie, N. Lichiardopol, P. Moree, P. Solé, On Robin's criterion for the Riemann hypothesis, Journal de Théorie des Nombres de Bordeaux 19 (2) (2007) 357-372. doi:doi:10.5802/jtnb.591.

[3] A. Hertlein, Robin's Inequality for New Families of Integers, Integers 18.

[4] J. B. Rosser, L. Schoenfeld, Approximate Formulas for Some Functions of Prime Numbers, Illinois Journal of Mathematics 6 (1) (1962) 64-94. doi:doi:10.1215/ijm/1255631807. 